Goldschmidt 2021 Abstract

https://doi.org/10.7185/gold2021.3990

\section{The future of coral reefs is a joint project}

\section{IGOR PESSOA, MAURO GERALDES AND LUZIA ANTONIOLI}

Rio de Janeiro State University

Presenting Author: pessoa.igor@gmail.com

In coral reef studies, mass spectrometry methods are widely applied to determine geochemical proxies in corals as a tool to evaluate seawater changes. Such procedure allows us to compare past and present ocean conditions. For example, the isotopic composition of oxygen in fossil corals reveals past ocean temperatures, the precise quantification of trace elements within the skeleton of deep-sea corals reveals past ocean circulation over geologic timescales, and the investigation of the carbon cycle in reefs quantifies the carbon budget of coral reef systems. This line of research has great value because we can obtain records of seawater parameters that can be used for the investigation of threats that affect the health of coral reefs. In this way, mass spectrometry methods enable an in-depth analysis of environmental issues relating to global warming and how they are connected with marine biogeochemical cycles. These analytical tools can lead to a better understanding of the mechanisms that govern the tolerance and vulnerability of corals to climate change. Given the current climate crisis, this issue requires accurate measurements to increase our understanding of the impacts that have become more frequent and intense.

To avoid the worst-case scenario, which may become the extinction of an entire ecosystem without direct coral focused intervention efforts. Strategies to increase our understanding on corals resistance and recovery are needed to address biodiversity security as the greatest emerging challenge. Additionally, science communication to raise awareness needs to include both, nonformal less scientific threads and science-based threads. Science communication turns scientific knowledge on threats to coral reefs into actionable information that can be exploited by a wide range of users, including public authorities, businesses and the public. The information allows the users to account for the effects of climate change into their decision making, as well as bring new opportunities to collaborate with better coral reef management through community-engaged research, citizen science, and regenerative tourism initiatives. Accordingly, the transmission of knowledge leads those who rely on healthy coral reefs to begin working together to protect the shared resources that are so important to tourism and food security.
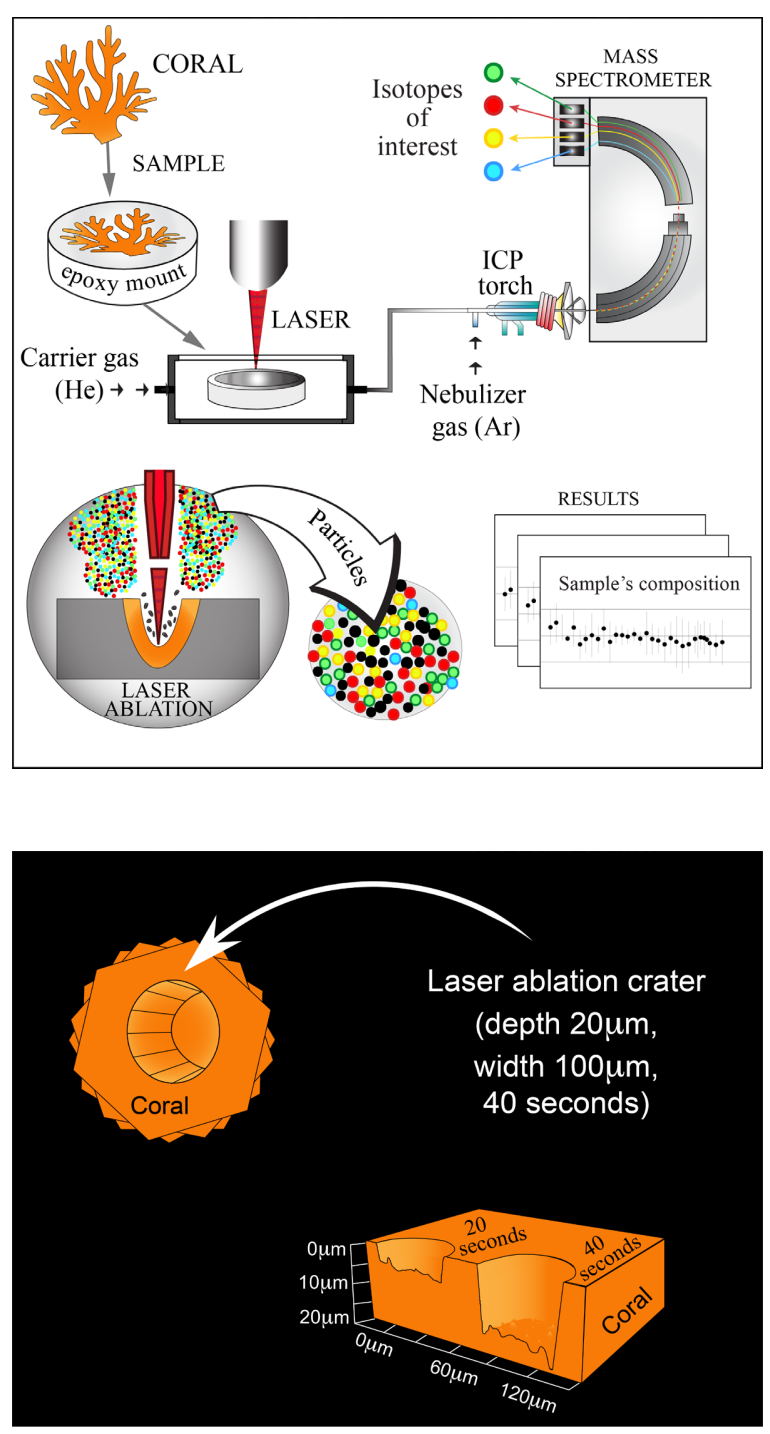

Video Abstract 